\title{
Recurrent vascular events in ischaemic stroke patients with diabetes
}

Narayanaswamy Venketasubramanian, ${ }^{1}$ FAMS (Neurology), MHlthSci (Stroke management) (Newcastle), MSc (Epidemiology) (London)

Stroke is a major cause of death and disability globally, with 6.55 million deaths $(95 \%$ uncertainty interval [UI] 6.00-7.02 million), 101 million prevalent cases (95\% UI 93.2-111 million) and 143 million disability adjusted life years lost (DALYs) (95\% UI 133-153 million) in 2019. ${ }^{1}$ The impact is even higher in Asia with its rapidly ageing populations and economies in transition, which will be associated with increasing incidence of cardiovascular risk factors. ${ }^{2}$ Among the many unfavourable outcomes after transient ischaemic attack or ischaemic stroke is recurrent vascular events. In a meta-analysis of 58 studies $(n=131,299)$ with a mean follow-up of 3.5 years (range 1.0-10.0), the risk of recurrent stroke was $4.26 \%$ year $(95 \%$ confidence interval [CI] 3.43-5.09), while the risk of MI was $1.67 \%$ year (95\% CI 1.36-1.98). ${ }^{3}$

The global prevalence of type 2 diabetes mellitus was $6,059 / 100,000$ in 2017 , with approximately 462 million individuals affected. ${ }^{4}$ Hyperglycaemia during the acute phase of stroke is associated with poorer functional outcomes. This finding has driven the recommendation to treat hyperglycaemia to achieve blood glucose levels in a range of $140-180 \mathrm{mg} / \mathrm{dL}$ $(7.8-10.0 \mathrm{mmol} / \mathrm{L})$, with insulin if needed. ${ }^{5}$ However, while there are recommendations for reducing vascular event recurrence, there is no secondary prevention strategy recommended for diabetics as compared to non-diabetics. Clear evidence of differing outcome risk in these dichotomised groups would spur research into targeted therapies, specifically within these strata.

The paper in this issue of the Annals by The et al. seeks to provide an answer to this important question. ${ }^{6}$ This is a single-centre prospective cohort study of consecutive patients with (presumably acute) ischaemic stroke who were recruited from the Stroke Unit of the National University Hospital, Singapore. Patient demographics were collected and investigations performed in a standardised manner. Diabetes was diagnosed using the American Diabetes Association recommendations: either fasting glucose $\geq 7.0 \mathrm{mmol} / \mathrm{L}$, 2-hour oral glucose tolerance test $\geq 11.1 \mathrm{mmol} / \mathrm{L}$ or HbA1c $\geq 6.5 \%$. Diabetes was considered "newly diagnosed" if diagnosed within 3 months of stroke onset, and "pre-existing" if diagnosed more than 3 months prior to stroke onset. It is unclear what proportion were diagnosed by the investigators as having diabetes mellitus after the stroke-hyperglycaemia during acute stroke is usually seen as a stress response, and the performance of an oral glucose tolerance test at this time point may not be appropriate; a raised HbAlc may however be a more suitable diagnostic test for diabetes mellitus in such a setting. ${ }^{7}$ Nonetheless, the high $(43 \%)$ frequency of diabetes mellitus in the study cohort is consistent with other Asian stroke registries, as mentioned by the investigators. Of note is that $30 \%$ of the diabetics were newly diagnosed, which may indicate their under-diagnosis in the community.

At baseline, there was no significant difference in age or gender between the diabetics and non-diabetics, but there was a higher frequency among diabetics of nonChinese ethnicity. As expected, overweight/obesity, hypertension and hyperlipidaemia consistent with the metabolic syndrome, as well as other prior vascular events such as coronary and peripheral artery disease, had a non-significant higher frequency prior stroke. Stroke mechanisms were however surprisingly not significantly different between diabetics and nondiabetics - as mentioned by the investigators, a high frequency of large artery atherosclerosis or small artery occlusion would be expected as reported by other authors and maybe more atrial fibrillation. The investigators attributed their finding to better glycaemic control of their patients. Another explanation may be related to the researchers' decision to exclude those who may die in a month after enrolment (the deceased contributed to the $12.8 \%$ excluded) - these are likely to have been severe strokes, usually due to large artery atherosclerosis; the median National Institutes of Health Stroke Scale of study participants was a very low 2 (interquartile range [IQR] 1-4).

During the median follow-up of 3.25 years (IQR 1.084.67), the investigators detected overall 6.90 events per 1,000 person-month - cardiovascular events comprised 133 cerebrovascular (29 fatal stroke, 85 non-fatal stroke

${ }^{1}$ Raffles Neuroscience Centre, Raffles Hospital, Singapore

Correspondence: Dr Narayanaswamy Venketasubramanian, Raffles Neuroscience Centre, Raffles Hospital, 585 North Bridge Road, Singapore 188770.

Email: ramani_nv@rafflesmedical.com 
and 19 transient ischaemic attacks) and 42 coronary artery events (5 fatal myocardial infarction and 37 non-fatal myocardial infarction/unstable angina). Recurrent vascular events among patients with symptomatic cerebrovascular disease were more often in the cerebrovascular bed than cardiovascular bed, similar to what was seen among the 18,189 ischaemic stroke patients in the international REduction of Atherothrombosis for Continued Health (REACH) registry that had a large number of Asian patients. ${ }^{8}$

The primary finding of this paper is that ischaemic stroke patients with diabetes mellitus have a higher risk of recurrent vascular events than non-diabetics (adjusted hazard ratio of diabetes was 1.50 [95\% CI 1.08-2.10]). While this issue was not expressly discussed by the investigators, of relevance is that among the 14,526 ischaemic stroke patients in the China National Stroke Registry, there were higher frequencies of recurrent stroke at the 3-and 6-month time points among diabetics compared to non-diabetics. ${ }^{9}$ Similarly, diabetes mellitus was associated with increased risks of death, cardiovascular and non-cardiovascular hospitalisations, heart failure and ischaemic stroke/transient ischaemic attack recurrence compared to non-diabetics in a US nationwide stroke registry of ischaemic stroke patients aged $\geq 65$ years $(n=409,060) .{ }^{10}$

The novel finding in this paper is that even after adjusting for other confounders, Malay and Indian ethnicities were identified as independent predictors of recurrent vascular events compared to Chinese. The reason for this is unclear and a fertile ground for further research. Another interesting finding is that while BMI $<23 \mathrm{~kg} / \mathrm{m}^{2}$ was a predictor of recurrent vascular events, contrary to this is the still inexplicable "obesity paradox" where obesity was found to be associated with reduced recurrent stroke risk. ${ }^{11}$ The investigators hypothesise on a possible role of malnutrition in increasing the likelihood of recurrent vascular events among their patients; however, they found no effect of central obesity. Perhaps other anthropometric indices of obesity may be helpful.

This insightful paper supports the clinical concern that diabetics are at increased risk of recurrent vascular events compared to non-diabetics, with specific data for patients with ischaemic stroke. That there are also higher recurrent vascular risks among diabetics of Malay and Indian ethnicities compared to Chinese, pose additional concerns for healthcare in our multiethnic society. The authors are justified in highlighting implications to the design of future interventional studies.

\section{REFERENCES}

1. Roth GA, Mensah GA, Johnson CO, et al. Global Burden of Cardiovascular Diseases and Risk Factors, 1990-2019: Update From the GBD 2019 Study. J Am Coll Cardiol 2020;76:2982-3021.

2. Venketasubramanian N, Yoon BW, Pandian J, et al. Stroke Epidemiology in South, East, and South-East Asia: A Review. J Stroke 2017;19:286-94.

3. Boulanger M, Béjot Y, Rothwell PM, et al. Long-Term Risk of Myocardial Infarction Compared to Recurrent Stroke After Transient Ischemic Attack and Ischemic Stroke: Systematic Review and Meta-Analysis. J Am Heart Assoc 2018;7:e007267.

4. Khan MAB, Hashim MJ, King JK, et al. Epidemiology of Type 2 Diabetes - Global Burden of Disease and Forecasted Trends. J Epidemiol Glob Health 2020;10:107-11.

5. Powers WJ, Rabinstein AA, Ackerson T, et al. Guidelines for the Early Management of Patients With Acute Ischemic Stroke: 2019 Update to the 2018 Guidelines for the Early Management of Acute Ischemic Stroke: A Guideline for Healthcare Professionals From the American Heart Association/American Stroke Association. Stroke 2019;50:e344-418.

6. The EZ, Ng MY, Ng GJL, et al. Long-term outcomes of ischaemic stroke patients with diabetes in a multi-ethnic cohort in Singapore. Ann Acad Med Singap 2020;50:16-25.

7. Roquer J, Rodríguez-Campello A, Cuadrado-Godia E, et al. The role of $\mathrm{HbAlc}$ determination in detecting unknown glucose disturbances in ischemic stroke. PLoS One 2014;9:e109960.

8. Venketasubramanian N, Röther J, Bhatt DL, et al. Two-year vascular event rates in patients with symptomatic cerebrovascular disease: the REACH registry. Cerebrovasc Dis 2011;32:254-60.

9. Jia Q, Zhao X, Wang C, et al. Diabetes and poor outcomes within 6 months after acute ischemic stroke: the China National Stroke Registry. Stroke 2011;42:2758-62.

10. Echouffo-Tcheugui JB, Xu H, Matsouaka RA, et al. Diabetes and long-term outcomes of ischaemic stroke: findings from Get With The Guidelines-Stroke. Eur Heart J 2018;39:2376-86.

11. Andersen KK, Olsen TS. The obesity paradox in stroke: lower mortality and lower risk of readmission for recurrent stroke in obese stroke patients. Int J Stroke 2015;10:99-104. 